\title{
Future of Antiproton Triggered Fusion Propulsion
}

\author{
Brice N. Cassenti ${ }^{*}$ and Terry Kammash** \\ University of Connecticut, Storrs, CT and University of Michigan, Ann Arbor, MI
}

\begin{abstract}
Antiproton triggered fusion propulsion appears to be a promising method for achieving high specific impulse and high thrust in a nuclear pulse propulsion system. In antiproton triggered fusion systems the antiprotons are injected into a pellet containing fusion fuel with a small amount of fissionable material. The fission fragments resulting from the annihilation of antiprotons are used to trigger a fusion reaction. Initial estimates indicate that if magnetically insulated inertial confinement fusion is used that the pellets should result in a specific impulse of between 100,000 and 300,000 seconds at high thrust. The engineering problems that must be overcome are significant. Among the challenges the most difficult may be the precise focusing of the antiprotons required to generate extremely large magnetic fields. Other challenges include the pellet design necessary to contain the fission and initial fusion products which will also require strong magnetic fields. The fusion fuel must also be contained for a sufficiently long time to effectively release the fusion energy, and the payload must be shielded from the radiation, especially the excess neutrons emitted, in addition to other particles.
\end{abstract}

$\begin{array}{lll}\mathrm{H} & = & \text { Hydrogen } \\ \mathrm{He} & = & \text { Helium } \\ \mathrm{Li} & = & \text { Lithium } \\ \mathrm{Pa} & = & \text { Palladium } \\ \mathrm{N} & = & \text { Neutron } \\ \mathrm{U} & = & \text { Uranium } \\ X & = & \text { Fission Product } \\ \gamma & = & \text { Photon } \\ \pi & = & \text { Pion }\end{array}$

\section{Nomenclature}

\section{Introduction}

$\mathrm{O}$ ver the last fifty years nuclear fusion propulsion has been the subject of considerable research. Fusion propulsion promises an extremely high specific impulse $(100,000$ to $3,000,000$ seconds) with some versions possessing high thrust. Most of the research has considered using fusion power reactor designs as the basis for the propulsion system. An excellent summary can be found in Williams and Borowski ${ }^{1}$. The concepts have progressed to the point where design practice guidelines have been proposed for fusion propulsion systems ${ }^{2}$. Propulsion based on fusion reactor designs though typically have low thrust to mass ratios and hence low accelerations. The thrust to mass ratio would be significantly increased if pulse fusion propulsion systems, in the form of explosive pellets, were developed. The specific impulse would remain high and use could be made of nuclear weapons technology. The pellets though need not contain a critical mass of fissionable material and hence would satisfy the ban on nuclear weapons in space.

Pulse nuclear propulsion appears closer to reality than magnetic confinement fusion propulsion and with the possibility of high specific impulse together with high thrust it clearly requires serious study. Nuclear pulse propulsion has been the subject of research since the $1950 \mathrm{~s}^{3}$. These early concepts examined external pulse

\footnotetext{
${ }^{*}$ Professor in Residence, Mechanical Engineering, Associate Fellow AIAA

${ }^{* *}$ Professor Emeritus, Nuclear Engineering Department, Associate Fellow AIAA
} 
propulsion where small critical mass fission devices are ejected from the rear of the rocket. A pusher plate riding on shock absorbers is used to absorb some of the energy from the detonation. The original design called for the absorbed energy to ablate the surface of the pusher plate to provide the thrust for the rocket. Devices that are detonated in an enclosed chamber (i.e., internal pulse propulsion) were also considered ${ }^{3}$. For internal pulse propulsion, ablation was also considered an important process for developing the thrust.

During the 1990s there was renewed interest in external and internal pulse propulsion systems ${ }^{4,5,6}$. In these later pulse proposals, the nuclear devices considered are considerably smaller than a critical mass device. The new designs have pellet diameters in the range from $15 \mathrm{~cm}$ down to $2 \mathrm{~cm}$ in diameter. Detonations can occur using either inertial confinement fusion concepts ${ }^{1,7,8,9}$ or a high energy density trigger, such as antiprotons ${ }^{5}$. In inertial confinement fusion systems the compression can be provided using laser beams ${ }^{7}$ or particle beams ${ }^{8,9}$. Smith $^{5}$ has shown that it is more effective to annihilate antiprotons in a fissionable material, and then use the energy from the fission reaction to drive the fusion reaction in the pellet. In Smith's original proposal the fuel density was not sufficient to efficiently produce fusion and some compression was required (using ion beams). Combining antiprotons and transient magnetic fields, generated during the annihilation, may lead to a system that requires no compression or heating at all ${ }^{10}$. It is also possible that fissionable material could be used to boost the performance of a fusion propulsion system ${ }^{11}$.

Important nuclear reactions are reviewed before proceeding to a discussion of the future of nuclear pulse propulsion systems.

\section{Nuclear Reactions}

Tritium $\left({ }_{1} \mathrm{H}^{3}\right)$ and deuterium $\left({ }_{1} \mathrm{H}^{2}\right)$ is frequently considered in fusion power applications since it has the lowest ignition temperature of all fusion reactions. The reaction produces helium- 4 and a neutron $\left({ }_{0} \mathrm{n}^{1}\right)$ according to

$$
{ }_{1} H^{2}+{ }_{1} H^{3} \rightarrow{ }_{2} \mathrm{He}^{4}+{ }_{0} n^{1}
$$

Note that the subscripts are the atomic number (nuclear electric charge) and the superscripts are the number of nucleons (total of neutrons and protons). The superscripts and subscripts have the same sum on both sides of the reaction for strong and electromagnetic interactions. The helium nucleus carries off $3.5 \mathrm{MeV}$ in kinetic energy, and since it is charged it can be used for propulsion, by either directing it with electromagnetic fields or by using it to heat a propellant. The neutron carries off $14 \mathrm{MeV}$ in kinetic energy, but the neutron can neither be readily directed by electromagnetic fields, nor can its energy be easily absorbed.

The $14 \mathrm{MeV}$ neutron is a radiation hazard that will need to be dealt with but it can also be used to split uranium or plutonium nuclei. This fission reaction can be represented, for example, as

$$
{ }_{0} n^{1}+{ }_{92} U^{238} \rightarrow{ }_{k_{1}} X^{N_{1}}+{ }_{k_{2}} X^{N_{2}}+a_{0} n^{1}+\gamma
$$

where $\mathrm{X}$ represents fission fragments. These fragments carry off more than $140 \mathrm{MeV}$ in kinetic energy. The neutrons emitted each have an average kinetic energy of about $2.1 \mathrm{MeV}$. An average of 3.8 neutrons are emitted (i.e., $a_{0}$ is about 3.8). Note that in the above $k_{1}+k_{2}$ is 92 and $N_{1}+N_{2}+a_{0}$ is 239. Photons, $\gamma$, in the form of X-rays carry off the remaining energy released in the reaction. The neutrons emitted in this first level fission reaction can induce second level fission reactions, but now the number of neutrons emitted is smaller. The reaction can still be written as

$$
{ }_{0} n^{1}+{ }_{92} U^{238} \rightarrow{ }_{k_{3}} X^{N_{3}}+{ }_{k_{4}} X^{N_{4}}+b_{0} n^{1}+\gamma
$$

For this lower energy reaction two nuclear fragments are emitted, as before, but now $b_{0}$ is about 2.7. It is important to note that in reactions (2) and (3) the nuclear fragments are usually neutron rich and, hence, radioactive.

The neutrons emitted by the fission or the fusion reaction can also be used to breed tritium. When lithium- 6 absorbs a neutron it has a high probability of splitting into a tritium nucleus and a helium-4 nucleus according to 


$$
{ }_{0} n^{1}+{ }_{3} \mathrm{Li}^{6} \rightarrow{ }_{2} \mathrm{He}^{4}+{ }_{1} \mathrm{H}^{3} \text {. }
$$

This reaction is useful since lithium-6 is easier to handle and store than tritium, but the added mass of the lithium nucleus tends to reduce the specific impulse of the pellet. Also the reaction cross-section is small which will require either larger pellets or compression. A more complete discussion of the use reactions (1) through (4) can be found in references 11 and 12.

In this paper we will also be considering antiproton annihilation in heavy nuclei. When antiprotons annihilate on the surface of a fissionable nucleus, such as uranium or plutonium, the nucleus splits into two lighter nuclei and many neutrons ${ }^{3}$. The energy from the mass annihilation of the antiproton and a nucleon (a proton or a neutron) in the nucleus is large enough to release between 12 and 18 neutrons. An important feature of the annihilation is that most of the fission fragments are not neutron rich and, hence, are not radioactive. The key idea is to use the kinetic energy of the fission fragments to heat the fusion fuel, and therefore the fission fragments must remain trapped long enough to transfer their kinetic energy.

If the antiproton annihilates against a proton in a U-238 nucleus the reaction can be written as

$$
{ }_{-1} H^{-1}+{ }_{92} U^{238} \rightarrow_{k_{5}} X^{N_{5}}+_{k_{6}} X^{N_{6}}+c_{0} n^{1}+n \pi^{+}+n \pi^{-}+m \pi^{0}+\gamma
$$

Generally pions $(\pi)$ are emitted in the annihilation and many of these are reabsorbed in the nuclear fragments. Note that the pions have zero for a nucleon number and are charged either positive $\left(\pi^{+}\right)$, negative $\left(\pi^{-}\right)$or neutral $\left(\pi^{0}\right)$. For this case that $c_{0}$ is between 12 and 18 , and the fission fragments are generally not radioactive ${ }^{5}$.

\section{Nuclear Pulse Fusion Propulsion}

Nuclear pulse propulsion has been proposed in various forms since the 1950s. Systems examined were: 1) critical mass pulse propulsion systems, 2) non-critical mass pulse propulsion systems and 3) contained pulse systems.

\section{A. Critical Mass Systems}

Nuclear pulse propulsion was first proposed by Stanislaus Ulam in $1946^{13}$. Ulam considered both contained explosions (an internal nuclear pulse propulsion system) and external pulse systems. By the mid-1950's it was clear that external pulses acting on a plate with shock absorbers would result in the best performance ${ }^{13}$. Of these, the Orion vehicle concept was developed in considerable detail ${ }^{14}$. A thorough technical discussion on Orion, with references, can be found in Martin and Bond ${ }^{8,9}$. Fission bombs were usually considered. The first few detonated would be small - around 0.1 kilotons. They would gradually increase in energy with the last having a yield of about 20 kilotons. The mass of the propulsion system was about 91 metric tons and the thrust was near $32 \times 10^{6} \mathrm{~N}$. The specific impulse averaged around 2500 seconds. But the specific impulse could have been as high as 10,000 seconds depending on the yield of the weapons and the design of the pusher plate.

Solem ${ }^{15,}{ }^{16}$ has recently proposed a variation in which the pusher plate and shock absorbers were replaced by a parachute and cords respectively. His vehicle design, Medusa, has a predicted specific impulse of 13,800 seconds to 43,500 seconds and average thrust-to-weight of 0.003 to 0.030 .

In both Medusa and Orion greater performance can be obtained by using critical mass fission devices to trigger a fusion reaction. There is little doubt that some version of critical mass pulse propulsion will work, but the ban on the use of nuclear weapons in space means that methods other than those using a critical mass for igniting the nuclear reactions must be found.

\section{B. Non-Critical Mass External Pulse System}

In 1978 the British Interplanetary Society completed a landmark study on an interstellar spacecraft to reach Barnard's at 6 light years from the sun ${ }^{8,9}$. The vehicle used a pulse fusion propulsion concept that did not require a critical mass of fissionable material. The vehicle consisted of two stages and used an inertial confinement fusion system for propulsion with deuterium-helium-3 pellets as the fuel. The pellets were compressed using relativistic 
electron beams. The specific impulse for each stage was close to one million seconds. The first stage fired for 2 years and the second for 1.75 years. The final velocity was 0.128 times the speed of light.

The final mass of the proposed design was 931 metric tons. The initial first stage mass was 50,000 metric tons, and the initial first stage thrust was $7 . \times 10^{6} \mathrm{~N}$. The second stage thrust was set at $0.66 \times 10^{6} \mathrm{~N}$. Most of power (about $700 \mathrm{GW}$ ) and a considerable mass (at least 140 tons) were needed for the compression subsystem. Only a preliminary weight and power study was conducted.

The Daedalus study considered a spacecraft propulsion system well beyond current technology. The primary problem was igniting the fusion reaction. For smaller velocity missions the compression system will occupy a larger fraction of the mass and power requirements, and, in fact, will dominate the vehicle mass. Alternatives to compression, without a critical mass of fissionable material present, would have a considerable impact on the system requirements and this remains the primary engineering challenge in inertial confinement fusion pulse propulsion. The Medusa vehicle solves the problem of the mass associated with the compression system and eliminates the need to use energy form the fusion reaction to drive the compression system, but cannot be considered since it is a critical mass system.

\section{Contained Systems}

Contained systems are a third class of nuclear pulse propulsion system and are conceptually easier to develop. They were dropped because of their poor performance ${ }^{2,}{ }^{13}$. Parlos and Metzger ${ }^{17}$ though resurrected the idea of contained nuclear pulse propulsion. They noted that a containment vessel, using newly developed materials, can be constructed that will have a sufficient fatigue life when a nuclear weapon is detonated inside. Their concept had a specific impulse of 1200 seconds and a thrust-to-weight ratio of up to ten. A later design ${ }^{18}$ demonstrates that it may be possible to design a contained system that can achieve a specific impulse of 3,800 seconds and a thrust-to-weight ratio of 30. Although advanced materials have significantly improved the performance of contained systems over the last forty years, they are, and will remain, the primary factor limiting the performance of internal pulse propulsion systems. The proposal by Parlos and Metzger use critical mass devices and hence does not satisfy the nuclear test ban treaty.

Hence we can conclude that solution to the problem of igniting a pellet without a critical mass of fissionable material and without the high mass and energy of external compression is the major impediment to making nuclear pulse propulsion a reality.

\section{Magnetically Insulated Inertial Confinement Fusion Systems}

Critical mass systems (internal or external) and compression systems both appear unlikely to be developed in the near future, but other technologies may be able to make fusion propulsion a reality. One of these is MICF (Magnetically insulated Inertial Confinement Fusion) first proposed by Kammash ${ }^{19}$, 20 In MICF propulsion containment is aided by intense transient magnet fields created when the surface of a solid is heated by a high power laser. At the focus ions and electrons will be emitted from the surface. The electrons, because of their small mass are moving considerably faster generating a net negative current flow for a very short time as illustrated in Figure 1. The magnetic fields can be as high as ten million gauss and will impede the flow of the plasma. If a fusion fuel is ionized then the temperature and density may be high enough, long enough, to initiate a fusion reaction.

Propulsion systems based on MICF can have a specific impulse as high as $175,000 \mathrm{sec}$ at low thrust. The vehicles would still be massive, and the laser, with its power system, would be a significant fraction of the mass of the vehicle.

In an effort to remove the mass of the laser system Kammash and Galbraith ${ }^{21}$ proposed using antiprotons to create the plasma instead of a high energy laser. Unfortunately, the energy transfer to the fusion fuel was inefficient, and a large mass of antiprotons was required. But the system did completely remove the mass associated with the laser heating and replace it with a considerably smaller mass of the antiproton containment system and the antiproton delivery system. 


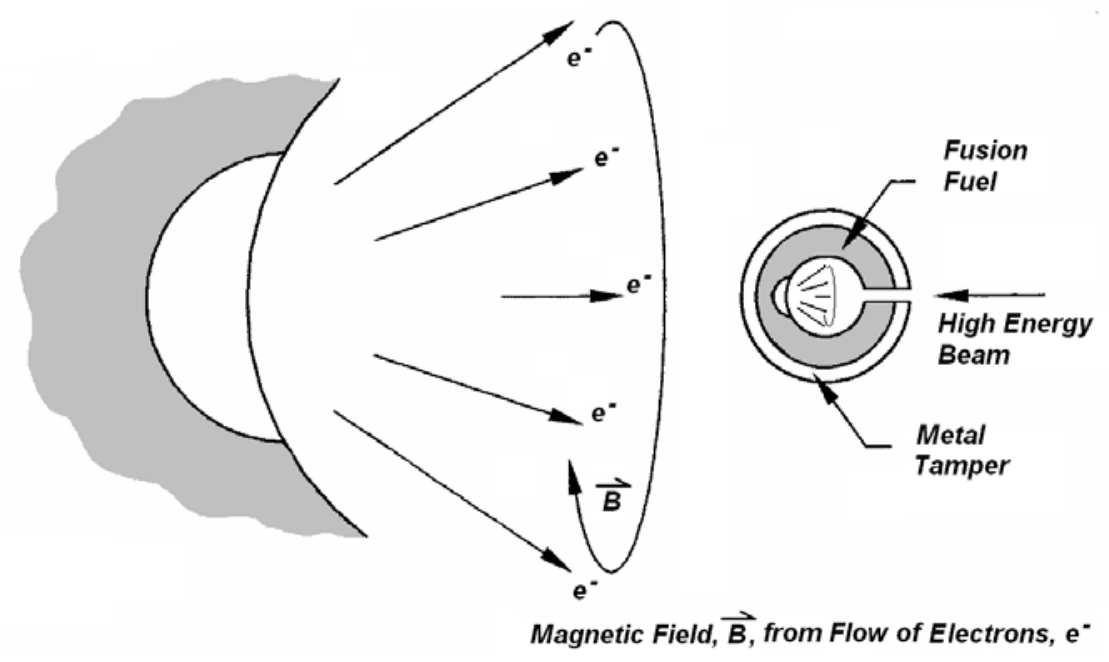

Fig. 1. MICF Plasma Containment ${ }^{10}$

A final problem with MICF is the radiation from the excess neutrons that will be produced in a tritium-deuterium reaction. Shielding will be required for the crew and sensitive electronic equipment. Ablation shields have been proposed as a possible solution, but probably will not provide any significant increase in the thrust ${ }^{22}$. In fact, analyses indicate ${ }^{22}$ that because the maximum temperature is below the surface of the shield (see Figure 2) the mass of shielding would be significantly larger than for a normal ablating re-entry vehicle since all the material from the maximum temperature to the surface will be lost. Hence, shielding the payload from the neutrons emitted in a deuterium-tritium system probably cannot be solved using ablation, and other solutions should be investigated.

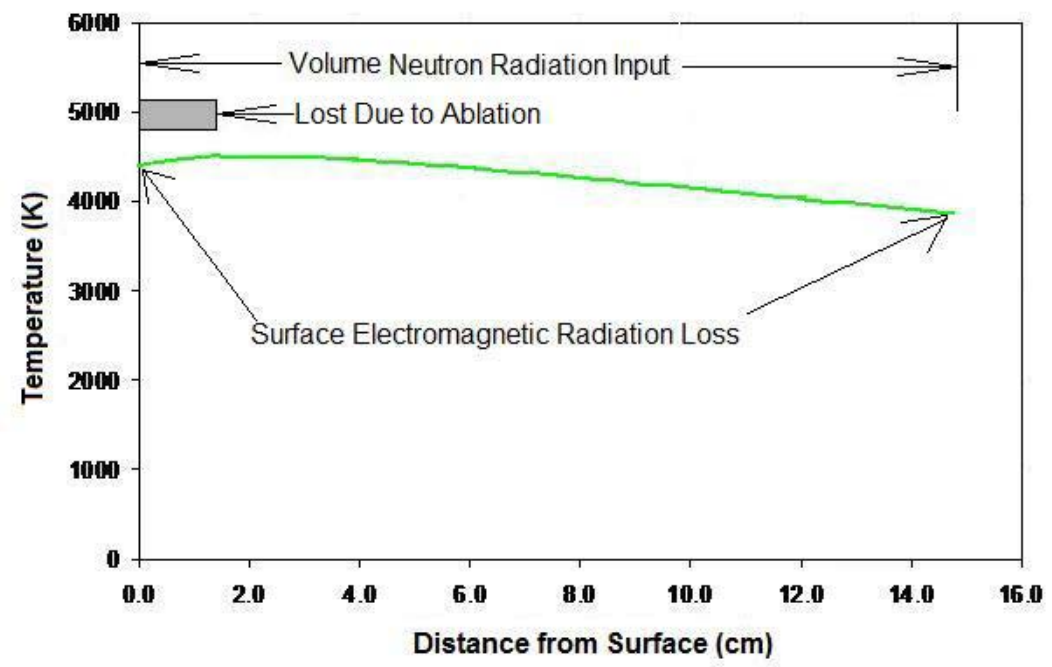

Fig. 2. Material Lost Due to Ablation (Adapted from Reference 22)

This problem also has a bearing on the use of ablation in external and internal nuclear pulse propulsion systems. Thrust cannot be provided by ablation since the absorbing material will come off in finite thickness segments and hence will move away at low velocity resulting in a low specific impulse. 


\section{Antiproton Triggered Systems}

In 1990 Gerry Smith and his colleagues at Pennsylvania State University ${ }^{5,23}$ described an ingenious proposal using antiprotons to initiate a sub-critical mass fission reaction. The antiprotons would be injected into a pellet containing plutonium and fusion fuel. Each annihilation reaction that occurs in the nucleus of a fissionable material will result a single nuclear fission reaction, but the products of the fission reaction could be used to heat fusion fuel.

A schematic of a pellet using antiprotons to initiate the fusion reaction is shown in Figure 3. One fission reaction is induced in the chip of U-235 for each antiproton injected. Very few additional fission reactions occur from the neutrons released in the fission ${ }^{12}$. The fusion fuel between the chip and the core is ionized and enters the core. The fusion reaction then proceeds through fusion fuel (deuterium and tritium in this case) to the outer shells of uranium and tungsten. These shells primarily provide mass to keep the fusion fuel contained long enough so that 5-10\% of the fusion fuel will react. For a pellet $2 \mathrm{~cm}$ in diameter and shells about $0.1 \mathrm{~mm}$ thick we estimate that between $5 \%$ and $10 \%$ of the fuel will react according the reaction (1). This would result in an ideal specific impulse of between 100,000 and 300,000 seconds $^{10}$. The uranium shell would not only provide inertia but would also occasionally split by absorbing the fusion neutrons to provide additional high speed particles to contribute to the specific impulse and to the compression of the fusion fuel.

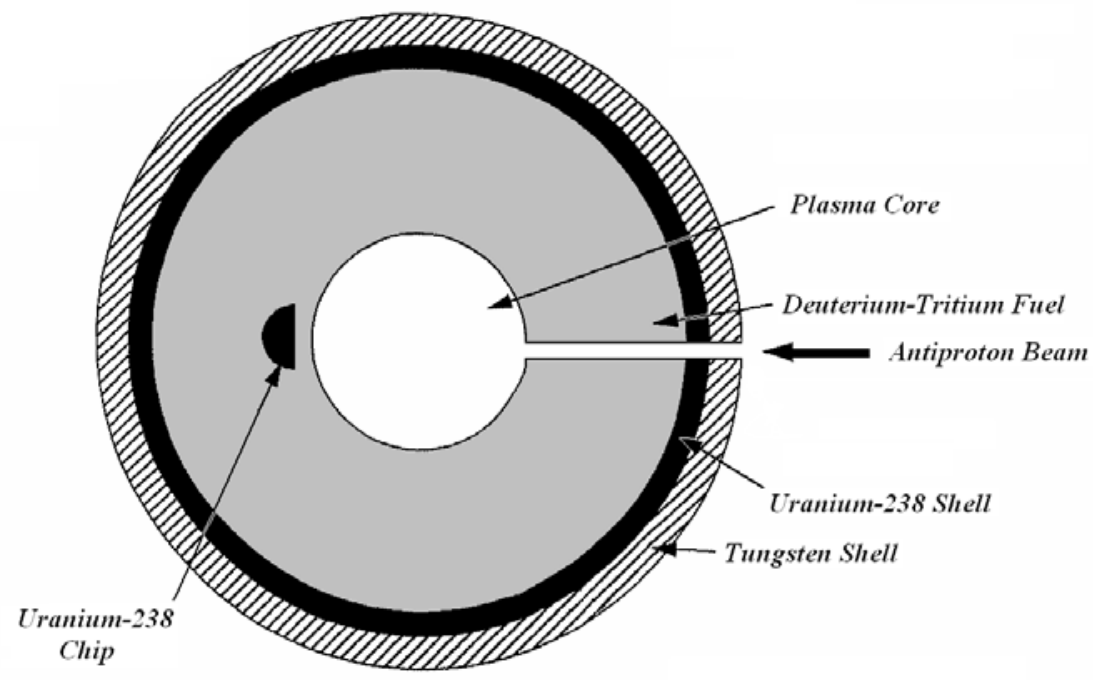

Fig. 3. Micro-fission MICF Fuel Pellet Construction ${ }^{12}$.

\section{Future Antiproton Triggered MICF Propulsion}

Combining the concept of MICF with the antiprotons to trigger a sub-critical mass fission reaction may remove any need to compress the pellets ${ }^{10}$ and result in a significant decrease in mass. Since, it is difficult to predict the exact extent of the fusion reaction, the pellet may have to be increased from a $2 \mathrm{~cm}$ diameter to possibly as large as $30 \mathrm{~cm}$. In order to determine the exact extent of the reaction it will be necessary to perform accurate plasma dynamics simulations. These simulations would also provide guidance for the development of a magnetic nozzle to direct the charged exhaust products. This approach may also require significant quantities of antiprotons (between $10^{11}$ and $10^{13}$ for each pellet), and, hence, more efficient antiproton production techniques may have to be developed than are currently available ${ }^{24}$.

The key to the performance of MICF is the generation of strong transient magnetic fields in the core of the pellet. The strength of these fields depends on the size of the spot where the annihilations occur, with a smaller spot producing larger field strengths. Hence the dispersion of the antiprotons as they enter the pellet is important. To keep the focus sharp without the need for the hole it should be possible to tune the incoming antiproton energy to a precise energy that will make the shells of metal and fuel nearly transparent to the injected antiprotons ${ }^{25}$, but accurate simulations or careful experiments need to be performed. Research needs to be performed to determine the transmission spectra of the pellet constituents to antiprotons for various injection energies for this technique to be used effectively. 
If the fusion of deuterium and tritium is considered, it may be advantageous to add fissionable material to use the neutrons given off in the fusion reaction ${ }^{11,12}$. By adding uranium-238 to the pellet the neutrons generated from the fusion of deuterium and tritium that are released will be sufficient to split the uranium. It should also be noted that the high density of uranium would also help to contain the explosion. Finally, it may be possible to use lithium-6 to generate tritium. This would completely remove the need to store the pellets at cryogenic temperatures. These additions would significantly increase the design space for pellet configurations. A schematic of such a pellet is shown n Figure 4. Note that the antiprotons are assumed to be tuned to a transmission energy and that the tamper is now uranium-238 with an explosive to help contain the plasma. The pellet in Figure 4 is more massive than the pellet in Figure 2 and hence would have a lower specific impulse unless the fuel fraction is increased.

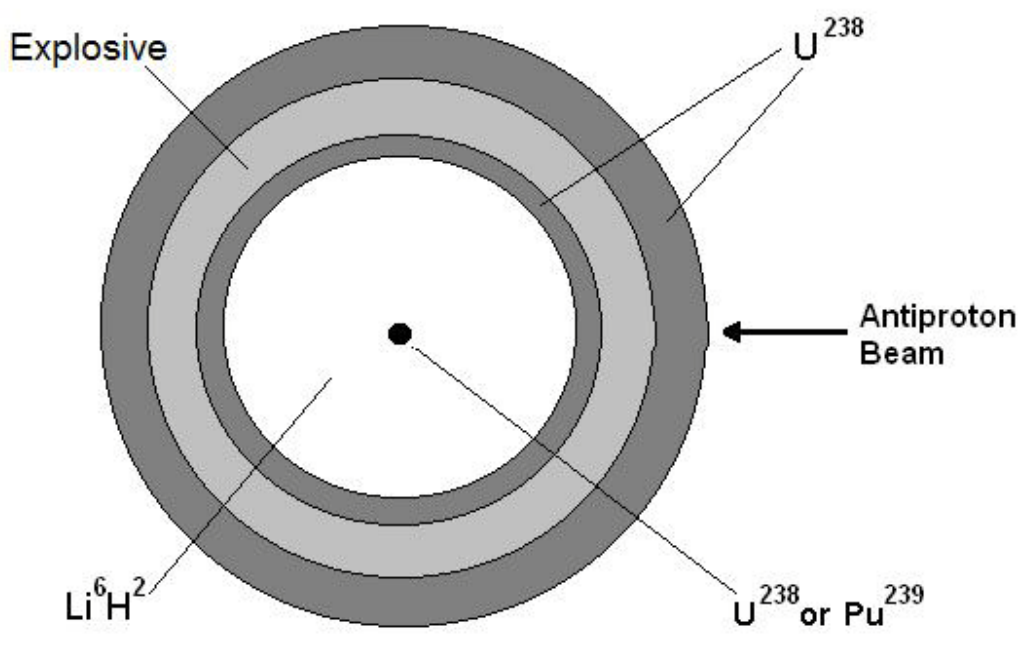

Fig. 4. Pellet Design using $\mathrm{Li}^{6} \mathrm{H}^{2}$ for Fusion Fuel (Adapted from Reference 12).

Analyses of hybrid pellet designs indicate that pellets on the order of $2 \mathrm{~cm}$ in diameter will probably require additional compression ${ }^{12}$, but sizes approaching $20 \mathrm{~cm}$ probably will not. Accurate simulations of the performance of a hybrid pellet will have to be performed to determine if there is any advantage to using lithium to generate tritium.

Finally there is the problem of neutron radiation damage. Shielding based on ablation will not be effective, but there are two possible solutions: 1) the neutrons could be used with lithium to generate the tritium as in a hybrid reaction, or 2) the higher energy fusion generated neutrons could be used to split a fissionable nucleus and turned into lower energy neutrons that require a smaller shielding mass.

\section{Conclusion}

Nuclear pulse propulsion holds the promise of high specific impulse with high thrust, but there are formidable engineering obstacles that must be overcome before it can become a reality. Pulse nuclear propulsion based on critical mass devices cannot satisfy the ban on nuclear weapons in space, but using nuclear weapons technology smaller devices that do not contain a critical mass of fissionable material can certainly be developed. Inertial confinement fusion using lasers or relativistic ion, or electron, beams for compression is one approach that does not depend on nuclear weapons technology, but the technology is complex and requires extremely accurate compression and extremely large power supplies. Much more important is the fact that the inertial compression system would dominate the mass of an inertial propulsion system. Magnetically insulated inertial confinement fusion (MICF) is a lightweight approach that could generate extremely high transient magnetic fields and these fields could be used to help to contain the fusion plasma. If lasers were used they would require large power supplies and hence would add a significant mass to he propulsion system, although it would be less than the mass requirements for an inertial 
confinement system. The mass of the lasers could be entirely removed if antiprotons were used to ignite a fusion reaction. The storage and delivery system would clearly be less massive than either a laser driven MICF system or a beam compression inertial confinement system. The annihilation energy generated can be effectively used if the antiproton is annihilated on a fissionable nucleus (e.g., uranium). The fission fragments could then be used to heat the fusion fuel. The problem here is the propagation of the fusion reaction through the pellet. It must last long enough for at least five percent of the fuel to fuse. This is the key question that must be answered. A second question is the size of the focal spot that will result from the injected antiprotons, which must remain small for sufficiently strong magnetic fields to be generated. It may be possible to precisely tune the antiproton energy to a transmission resonance so that the antiprotons annihilate precisely at a predetermined spot. Research needs to be performed to determine the transmission spectra of the pellet constituents to antiprotons. Finally there is the problem of neutron radiation damage. There are two possible solutions: 1) the neutrons could be used with lithium to generate the tritium or 2) the higher energy fusion generated neutrons could transformed into lower energy neutrons through fission that require a smaller shielding mass.

\section{Acknowledgment}

The authors wish to acknowledge the support of University of Connecticut for their help in preparation and presentation of this paper.

\section{References}

${ }^{1}$ Williams, C.H., and S.K. Borowski, "An assessment of fusion space propulsion concepts and desired operating parameters for fast solar system travel,” AIAA-1997-3074, 33rd AIAA/ASME/SAE/ASEE Joint Propulsion Conference and Exhibit, 33rd, Seattle, WA, July 6-9, 1997.

${ }^{2}$ Williams, C.H., "Application of Recommended Design Practices for Conceptual Nuclear Fusion Space Propulsion Systems," AIAA-2004-3534, 40th AIAA/ASME/SAE/ASEE Joint Propulsion Conference and Exhibit, Fort Lauderdale, Florida, July 11-14, 2004.

${ }^{3}$ Nance, J.C., "Nuclear Pulse Propulsion, ” IEEE Transactions on Nuclear Science, Vol. NS-12, pp. 177-182, 1965.

${ }^{4}$ Chwieroth, B. S., Lewis, R. A., Smith, G. A., et al, “Antiproton Catalyzed Microfission/Fusion Propulsion,” Paper AIAA 95-2900, AIAA/ASME//SAE/SEE Joint 31st Propulsion Conference San Diego, CA, 1995.

${ }^{5}$ Lewis, R., Newton, A. R., Smith, G. A., et al, "An Antiproton Catalyst for Inertial Confinement Fusion Propulsion,” AIAA/SAE/ASME/ASEE 26th Joint Propulsion Conference, Orlando, FL, 1990.

${ }^{6}$ Gaidos, G., Lewis, R.A., Meyer, K., et al, “AIMSTAR: Antimatter Initiated Microfusion for Pre-Cursor Interstellar Missions,” Paper AIAA 98-3404, AIAA/ASME/SAE/ASEE 34th Joint, Cleveland, OH, 1998.

${ }^{7}$ Kammash, T., and Galbraith, D. L., “Antimatter-Driven-Propulsion Systems for Solar system Exploration,” Journal of Propulsion and Power, Vol. 8, No. 3, pp. 644-649, 1992.

${ }^{8}$ Martin, A. R. and Bond, A., "Project Daedalus: The Propulsion System - Part 1: Theoretical Considerations and Calculations,” Journal of the British Interplanetary Society, Supplement Volume, pp. S44-S62, 1978.

${ }^{9}$ Martin, A. R. and Bond, A., "Project Daedalus: The Propulsion System - Part 2: Engineering Design Considerations and Calculations,” Journal of the British Interplanetary Society, Supplement Volume, pp. S63-S82, 1798.

${ }^{10}$ Cassenti, B. N., Kammash, T., and Galbraith, D. L., “Antiproton Catalyzed Fusion Propulsion for Interplanetary Missions,” AIAA Journal of Propulsion and Power, Vol. 13, pp.428-434, 1997.

${ }^{11}$ Cassenti, B. N., "Hybrid Fusion Propulsion," Paper 98-3590, 34th AIAA/ASME/SAE/ASEE Joint Propulsion Conference, Cleveland, OH, 1998.

${ }^{12}$ Cassenti, B. N., "Hybrid Fusion Propulsion, " Paper AIAA 99-2699, 35th AIAA/ASME/SAE/ASEE Joint Propulsion Conference, Los Angeles, CA, 1999.

${ }^{13}$ Martin, A. R. and Bond, A., "Nuclear Pulse Propulsion: A Historical Review of an Advanced Propulsion Concept,” Journal of the British Interplanetary Society, Vol. 32, pp. 283-310, 1979.

${ }^{14}$ McPhee, J., The Curve of Binding Energy, Farrar, Straus and Giroux, New York, 1974.

${ }^{15}$ Solem, J.C., "Medusa: Nuclear Explosive Propulsion for Interplanetary Travel," Journal of the British Interplanetary Society, Vol. 46, pp. 21-26, 1993. 
${ }^{16}$ Solem, J. C., "Nuclear Explosive Propulsion for Interplanetary Travel: Extension of the Medusa Concept for Higher Specific Impulse,” Journal of the British Interplanetary Society, Vol. 47, pp. 229-238, 1994.

${ }^{17}$ Parlos, A. G., and Metgzer, J. D., "Feasibility Study of a Contained Pulsed Nuclear Propulsion Engine,” Journal of Propulsion and Power, Vol. 10, No.2, pp. 269-278, 1992.

${ }^{18}$ Cassenti, B. N., “A Contained Antiproton Catalyzed Pulse Nuclear Propulsion System, ” Paper AIAA 95- 2898, 31st AIAA/ASME/SAE/ASEE Joint Propulsion Conference, San Diego, CA, 1995.

${ }^{19}$ Kammash, T. and Galbraith, D. L., “A Fusion Reactor For Spce Applications,” Fusion Technology, Vol. 12, pp. $11-21,1987$.

${ }^{20}$ Kammash, T. and Galbraith, D. L., "A High Gain Fusion Reactor Based on the Magnetically Insulated Inertial Confinement Fusion (MICF) Reactor,” Nuclear Fusion, Vol. 29, pp. 1079 -1099, 1989.

${ }^{21}$ Kammash, T. and Galbraith, D. L., "Antimatter Driven Fusion Propulsion System for Solar System Exploration," Journal of Propulsion and Power, Vol. 8, pp. 644-649, 1992.

${ }^{22}$ Cassenti, B.N.,“The Use of Ablation for Thrust in Pulsed Fusion Propulsion”, Paper AIAA 2003-4523 presented at the 39th AIAA/ASME/SAE/ASEE Joint Propulsion Conference and Exhibit, Huntsville, July 20-23, 2003.

${ }^{23}$ Lewis, R., Smith, G., Cardiff, R., et al, "Antiproton-Catalyzed Microfission/Fusion Propulsion Systems for Exploration of the Solar System and Beyond,” Paper AIAA 96-3069, AIAA/SAE/ASME 32nd Joint Propulsion lake Buena Vista, FL, 1996.

${ }^{24}$ Cassenti, B.N.,"Mass Production of Antimatter for High-Energy Propulsion" AIAA Journal of Propulsion and Power, 16, pp. 119-124, 2000.

${ }^{25}$ Cassenti, B.N.,“Antiproton Dispersion in Magnetically Insulated Inertial Confinement Fusion Propulsion”, Paper AIAA-2005-4135 presented at the 40th AIAA/ASME/SAE/ASEE Joint Propulsion Conference and Exhibit, Tucson, Arizona, July 10-13, 2005. 